\title{
Will We Ever Again Conduct in-Person Psychotherapy Sessions? Factors Associated with the Decision to Provide in-Person Therapy in the Age of COVID-19
}

\author{
Liat Shklarski ${ }^{1}$ (1) Allison Abrams ${ }^{2} \cdot$ Elana Bakst $^{2}$ \\ Accepted: 4 March 2021 / Published online: 13 March 2021 \\ (c) The Author(s), under exclusive licence to Springer Science+Business Media, LLC, part of Springer Nature 2021
}

\begin{abstract}
The COVID-19 pandemic has forced a new reality on the delivery of psychotherapeutic services. Therapists have had to rapidly adapt to telehealth therapy using various video conferencing technologies while working from spaces that were not necessarily designed for delivering therapy sessions. While COVID-19 continues to be present in therapists' lives and shapes how they provide services, answering the question of whether to meet with clients in person again is a decision laden with complexity and ambivalence. In this mixed-method study, surveys with 169 therapists and interviews with 17 therapists were used to determine the factors that currently influence their attitudes toward resuming traditional in-person psychotherapy in light of COVID-19. We focused on the therapists' personal and professional characteristics to assess their plans to continue with telepsychotherapy or return to a traditional psychotherapy setting. Our findings suggest that positive attitudes toward telepsychotherapy, the lack of an effective vaccine, clients' satisfaction with telepsychotherapy, insurance reimbursement for telepsychotherapy, and negative attitudes toward wearing masks during sessions influenced therapists' decisions on whether or not to return to traditional in-person psychotherapy in the age of COVID-19.
\end{abstract}

Keywords COVID-19 pandemic $\cdot$ In-person psychotherapy $\cdot$ Telepsychotherapy

The COVID-19 pandemic has had a significant and specific impact on the provision of mental health services in general and psychotherapy in particular. Therapists are adapting to conducting primarily remote therapy via video conferencing technologies and sometimes via telephone. Many are working from spaces that were not necessarily designed for conducting therapy sessions, such as their own homes, while others are seeing clients in their offices while wearing masks. Little is currently known about the effects of the COVID-19 pandemic on therapists, how the global pandemic is shaping their work with clients, and whether they will continue providing telehealth therapy or return to traditional in-person interactions with clients.

The growth of online therapy is well researched (Backhaus et al., 2012; Poletti et al., 2020; Roesler, 2017; Shigekawa et al., 2018), and in light of the COVID-19

Liat Shklarski

lshklars@ ramapo.edu

1 Ramapo College of New Jersey, Mahwah, USA

2 Hunter College, New York, NY, USA pandemic, we anticipate that many will come to realize the importance and the scope of teletherapy (Bell et al., 2020; Chherawala \& Gill, 2020; Vostanis \& Bell, 2020; Wind et al., 2020; Wright \& Caudill, 2020). We designed a mixedmethod study examining the factors that influence psychotherapists' decisions to return to the office to conduct in-person sessions during the COVID-19 pandemic as opposed to continuing to use telehealth methods to deliver psychotherapy. We focused on the therapists' attitudes toward remote work, demographics, and professional characteristics (such as work setup, modality of treatment, and population served) to assess their decisions to continue with telepsychotherapy or return to traditional in-person sessions. In this study, we examined how the global COVID-19 pandemic has affected therapists both personally and professionally and gathered data on their key considerations when faced with the prospect of returning to in-person therapy in light of COVID-19. 


\section{Literature Review}

Research on the provision of psychotherapy treatment during COVID-19 is emerging (Inchausti et al., 2020; Mitchell, 2020; Wright \& Caudill, 2020), but the majority has focused on the experiences of therapists with telehealth, the quality of remote therapy, and the working alliance during the pandemic. For example, Békés and Aafjes-van Doorn (2020) conducted a cross-sectional survey with 145 psychotherapists to assess their attitudes toward online psychotherapy at the beginning of the COVID-19 pandemic. The authors specifically compared between participants' previous attitudes toward online psychotherapy and their current reported experience. The results showed that for $67.6 \%$ of the participants, adjustment was made easier when they reported having their colleagues' professional support, and for $60 \%$, reading online posts helped with the transition. In addition, psychotherapists who reported positive experiences with online psychotherapy pre-pandemic and identified as cognitive-behavioral therapists had more positive attitudes toward telepsychotherapy when compared with those who conducted psychodynamic psychotherapy.

Humer et al. (2020) surveyed 338 psychotherapists from three European countries: Czech Republic $(n=112)$; Germany $(n=130)$, and Slovakia $(n=96)$ about their experiences with teletherapy and with having direct contact with clients during the COVID-19 pandemic. Data were collected during the month of May 2020, which was two weeks after the lockdown restrictions were lifted in the European countries. The results showed that although face-to-face psychotherapy was reduced and remote psychotherapy increased (both by phone and internet), face-toface psychotherapy remained the preferred method despite COVID-19. More specifically, German psychotherapists showed the lowest decrease in face-to-face psychotherapy. This may be explained by the fact that male participants from Germany reported having the least amount of fear in terms of conducting face-to-face psychotherapy during COVID-19 and health insurance companies in Germany (as opposed to in the Czech Republic and Slovakia) stopped paying for teletherapy in June 2020.

Feijt et al. (2020) qualitatively explored the experiences of 51 therapists from the Netherlands regarding telepsychotherapy during the COVID-19 pandemic using an online survey. Data were collected from April 1 to May 5, 2020. Similar to earlier findings, the practitioners reported having positive experiences with online treatment. Specifically, they highlighted that telepsychotherapy is convenient for the client and/or the therapist (e.g., because there is no travel time, scheduling is more flexible, and there is increased efficiency in administrative tasks and team meetings). The findings also showed that telepsychotherapy worked sufficiently well and that clients were satisfied.

Aafjes-van Doorn et al. (2020) surveyed the perceptions of 141 therapists toward the therapeutic relationship (the working alliance and the real relationship) when engaging in teletherapy sessions as compared to previous in-person therapy, along with therapists' confidence in their ability to provide teletherapy. Data for this study were collected in mid- to late March 2020. While the participants had relatively positive attitudes toward teletherapy $(\mathrm{M}=2.43$, $\mathrm{SD}=0.54$, range: $1.00-3.00$ ), they were largely undecided as to whether they planned to use teletherapy in the future. The findings showed that the therapists had experienced challenges in transitioning to remote therapy, but they felt that when using teletherapy sessions, their clients had relatively good experiences and relatively strong real relationships, similar to the levels reported for in-person therapy.

Békés (2020) surveyed the experiences of 190 psychoanalytically oriented therapists in their transition to teletherapy during the pandemic and their previous experiences with remote therapy. The findings showed that most participants $(n=122 ; 64.2 \%)$ adjusted well to the remote work and felt authentic in their work but experienced some difficulty with its technical aspects. While the results from this study also showed that the boundaries of the treatment changed, specifically at the beginning of the pandemic, the participants reported that their relationships with most of their clients during the pandemic felt as authentic as they had in their inperson sessions. In addition, pre-pandemic, the participants perceived online therapy as less effective. However, during the pandemic, they reported that teletherapy can be as effective as in-person treatment.

\section{Method}

\section{Procedure}

Upon receiving ethical approval from the Human Research and Ethics Committee of Ramapo College of New Jersey (IRB Protocol \#585), we recruited 169 mental health professionals through three dedicated professional listservs (Nonaffiliated with organizations) designed for social workers, psychologists, and art therapists. During the month of September 2020, we sent out a recruitment email that included a link to the survey. All participants signed an electronic informed consent form that included a statement of the ethics approval for the study as well as the goals of the research. One hundred and sixty-nine therapists agreed to participate in an online survey that included demographic questions as well as questions related to their decisions to continue with telepsychotherapy sessions or return to in-person sessions. At the end of the online survey, the participants were given 
the option of participating in a semi-structured interview. Each participant who provided their email address received a separate email in response inviting them to an interview. A total of 17 therapists participated in a telephone interview with the researchers.

\section{Instrument}

For the quantitative phase of the study, we developed an anonymous online survey with 43 questions to answer the research questions. The survey was designed following the recent literature (Feijt et al., 2020; Geller, 2020; Inchausti et al., 2020; Mitchell, 2020) and a content analysis of the email messages posted on psychotherapy listservs. For example: I have asked my office landlord for rent reductions, and what will make you feel safe for yourself and your patients in order to return to the office? In the survey, we used Likert scale statements (e.g., strongly disagree, disagree, neutral, agree, and strongly agree) as options for answering the questions. Higher scores on each scale indicated greater agreement with the questions present in each scale. The survey included positively and negatively worded questions that were reversed during the analysis to increase consistency.

For the qualitative phase of the study, we developed an interview guide with 10 questions that were based on the recent literature and empirical data on remote therapy during the pandemic (Geller, 2020; Inchausti et al., 2020; Mitchell, 2020). Phone interviews were conducted by the research team using a protocol that began by giving the participants an introduction to the study and was followed by asking the participants open-ended questions relating to their experiences with telepsychotherapy and their reasons for returning to the office to resume in-person sessions.

\section{Data Analysis}

Statistical Package for the Social Sciences (v. 27) was used to analyze the quantitative data. Reverse coding ensured that all the numerical scoring scales were in the same direction. The data were descriptively analyzed in order to demonstrate the shape, central tendency, and variability within the dataset. The results section presents the valid percentage of the responses.

We used bivariate Pearson correlations to assess the associations among continuous variables. A $p$-value of less than 0.05 was considered statistically significant. Independent samples $t$-tests and one-way ANOVA were conducted to assess group differences on continuous variables.

Following Braun and Clarke (2006), a thematic analysis was undertaken to analyze the qualitative data. In this process, the recorded interviews were transcribed and read in order to identify concepts. After repeating these steps for all the transcripts, emergent codes and categories were compared by two reviewers (the authors), connected as appropriate, and organized into key concepts.

\section{Results}

\section{Psychotherapists' Characteristics}

The majority of the participants self-identified as white women with an average age of $52(\mathrm{SD}=14.0$; range, 25-75); $63.3 \%$ had a degree in social work $(n=107), 20.1 \%$ held a psychology degree $(n=34), 13.5 \%$ had a mental health counseling degree $(n=23)$, and 3\% were psychiatrists $(n=5)$. The vast majority of the participants primarily worked with adults $(91.2 \%, n=154)$. In terms of the preferred modality of treatment, $67 \%$ of the participants $(n=113)$ used psychodynamic therapy, $17.1 \%$ used cognitive behavioral therapy $(n=29)$, and $15.9 \%$ of the participants $(n=27)$ used eclectic approaches including play therapy, trauma therapy, eye movement desensitization and reprocessing, and medication management. No statistically significant differences were found between the different professionals (social workers, psychologists and mental health counselors).

\section{Psychotherapy during the COVID-19 Pandemic}

Of the participants, $81.7 \%(n=138)$ had only seen clients remotely since the pandemic started in mid-March 2020. Of the $18.3 \%$ who had returned to the office, the majority reported that they had gone back during the month of August 2020. The results showed that $72.8 \%$ of the participants ( $n=123)$ had not returned to the office at all (i.e., they were still working from a location other than their office). Twenty-nine percent of the participants had asked their landlord to reduce their office rent or to break their office lease $(n=49)$. In addition, $71.6 \%$ of the participants had remained living in their main residence during the pandemic and were still living there at the time of the data collection $(n=121)$. Eighty-seven percent of participants $(n=147)$ lived with at least one additional person.

While the participants were conflicted about seeing clients in person, they also had some mixed feelings about working remotely from their domestic spaces. Participant ML explained:

I don't do my sessions at home any more, I found it too "challenging" in a two-bedroom apartment with my son and husband there to be able to focus on work; I tried it for a short while, but it was uncomfortable. I think it was confusing for my son.

This quote reflects a minority of participants' feelings of discomfort with working from home. In contrast, $81.1 \%$ of 
the participants $(n=137)$ agreed with the statement: "I am comfortable working from home."

Since the pandemic started, the participants stated that their attitudes toward remote psychotherapy had changed, and $60.4 \%$ reported that they prefered it more $(n=102)$. However, $70 \%$ of the participants $(n=103)$ agreed that remote work is more draining than in-person psychotherapy. To provide remote therapy, $47.9 \%$ of the participants $(n=81)$ used video platforms, $3 \%(n=5)$ used only the phone, and $49.1 \%(n=83)$ used a combination of both phone and video.

The vast majority of the participants $(88.2 \%, n=149)$ reported that in comparison to before the pandemic, they now have a positive attitude toward telepsychotherapy. In addition, $78.1 \%$ of the participants $(n=132)$ agreed with the statement: "Remote therapy can be as effective as inperson therapy." The qualitative inquiry provided more detailed information on their experiences. As participant TB explained: "I think the shape is very different, but I wouldn't qualify it as lesser or more. It's not quantitative. It's just very different but equally rewarding." This quote supports previous research (Békés \& Aafjes-van Doorn, 2020; Poletti et al., 2020; Roesler, 2017) showing that therapists perceive remote work to be as effective as in-person work; however, it requires certain shifts to be made in the approach such as the time required to build the therapeutic alliance, maintaining eye contact, and checking in with clients more frequently. To that end, $62.7 \%$ of the participants $(n=106)$ stated that once COVID-19 is no longer a risk, they will provide both inperson and telepsychotherapy services. Only $29 \%$ reported that they will only conduct face-to-face sessions $(n=49)$.

When asked for their thoughts on when they would feel comfortable returning to their office to conduct in-person therapy again, participant $\mathrm{AD}$ explained:

When [COVID] is no longer a contagious disease or that we're no longer in this pandemic... and I don't know what that would look like because you don't hear of diseases just disappearing, so to be honest, I don't know when I'll ever return to the office.

This quote reflects the conflicts that many of the participants seemed to be facing when thinking about the future of their practices. As stated earlier, many of the participants are still not seeing clients in person and do not currently work from their offices.

\section{Factors Associated with the Decision to Return to the Office to Provide in-Person Therapy}

When asked whether they were planning to return to the office in the foreseeable future, $75.8 \%$ of the participants $(n=128)$ responded that they were not. Unsurprisingly, $75.1 \%$ of the participants $(n=127)$ agreed with the statement: "I don't consider it safe for me or my clients to be traveling to the office and navigating public transportation, elevators, and bathrooms on a daily/weekly basis at the moment." The following factors were associated with participants' decisions to return to the office to conduct inperson sessions.

\section{Clients' Preferences}

The client's preference to be seen in person was associated with the decision to get back to the office. Participant NG explained that the decision to return was to some degree related to meeting her clients' needs:

I can't say that I missed the in-person work. But I do have one or two clients that have said that they would prefer to go back to the office, and so I have said when it is safe again, I will have maybe a day or a half day.

She continued: "But the majority of my clients are stating they're not interested, so it's probably not going to happen. So I don't even think I would rent an office full-time ever again."

About half of the participants reported that their clients had already asked them about the timeline to return to inperson treatment $(53.4 \%, n=91)$. Participants whose clients had asked them to be seen in person reported that they were more likely to return to the office before there is a vaccine in comparison to those whose clients had not asked them to be seen in person $(t=-5.61, d f=164.21, p<0.001)$. Similarly, participants' rating of the statement: "I am not planning to return to the office for the foreseeable future" was lower when their clients had asked them to be seen in person in comparison to participants whose clients had not asked about in-person sessions $(t=4.92, d f=158.62, p<0.001)$.

\section{Therapists' Preferences}

There was a positive association between the participants' agreement with the statement "I am comfortable working from home" and their agreement with the statement "I am not planning to return to the office in the foreseeable future" $(r=0.452, p<0.001)$. Similarly, a positive association was found between the question: "Now that you have already worked remotely for the past few months, do you believe that remote therapy can be as effective as in-person therapy?" and the statement "I am not planning to return to the office in the foreseeable future" $(r=0.489, p<0.001)$.

The quantitative data showed that $81.1 \%$ of the participants reported being comfortable working from home $(n=137)$. However, the qualitative data showed that attitudes among the participants varied greatly in regard to returning to in-person work versus continuing to work remotely. While some expressed their satisfaction with remote work, others felt quite differently, like participant LK: 
I think it degrades our profession if they're not coming into the office. It's a great support when needed, as a way to provide another avenue, but not to be able to interact physically - with couples in particular. You're getting an artificial sense of your clients. For me, the physical [is important]. I'm constantly looking at bodies and how they move in space. I'm not getting all the information I need as a clinician.

In addition, $56.8 \%$ of the participants $(n=96)$ reported that either they or someone close to them had health issues that would put them in a risk category for contracting COVID-19. Some of the participants reported that they have to be careful before they meet clients in person, mainly because of their family members whom they need to protect. For example, participant JK, who described himself as being in three different high-risk categories, stated that "there would have to be a huge shift in the disease profile for me to have clients back in the office." What that shift would look like was not entirely clear, but some considerations he listed included a "huge reduction in numbers" and "some effective treatment or vaccine. At least something as effective as the flu vaccine. Not necessarily a cure, but greatly reduced cases."

While there were no statistically significant findings related to participants' health conditions and their decision to return to seeing clients in person $(t=1.46, d f=140.49$, $p=0.147$ ), in the interviews, 10 participants raised their concerns for their own health and the health of their loved ones.

While some of the participants found working from home to be a convenient solution, the data also show that $61 \%$ of the participants $(n=103)$ reported that telepsychotherapy is more physically/cognitively/emotionally draining than in-person work, whereas $23.7 \%$ reported no differences $(n=40)$. Participant RB explained: “I'm much more tired at the end of the day [working remotely]... I feel like I'm putting more time and energy into seeing clients... it's something about staring into the screen so intently, whereas in person, it feels more relaxed."

The participants had mixed attitudes toward in-person treatment in light of the COVID-19 pandemic. Particularly participants who worked with children expressed a sense of urgency to return to meeting with clients face to face. Participant NG explained:

The idea of returning to in-person work makes me really nervous, but it's something I've been grappling with more and more particularly when it comes to children... Like my kiddos, some of them are really hard to engage over the phone if they don't have the appropriate devices or internet capabilities to do virtual sessions.
The challenge of working remotely with children has been addressed in previous research (Shklarski et al., 2021) and can be considered a reason for therapists to return to seeing their young clients face to face. The participants who reported working with children and adolescents agreed less with the statement: "Remote therapy can be as effective as in-person therapy" than the participants who worked with adults and families $(F(2,165)=6.95, p=0.003)$.

Finally, the majority of the participants $(88.7 \%, n=150)$ stated that they would not feel safe if a client started coughing during an in-person session. Over half of the participants stated that they experience some stress or anxiety when they think about seeing clients in person $(58 \%, n=98)$. There was a negative association between these statements $(r=-0.433$, $p<0.001)$, meaning that the participants' anxiety will increase if their clients cough during in-person sessions. Finally, there was a positive association between participants' affirmative response to the question: "If you return to the office before there is a proven vaccine, will you require your clients to wear masks?" and the statement "I experience some stress or anxiety when I think about seeing clients in person" $(r=0.415, p<0.001)$.

\section{Effective Vaccine}

When asked to answer the question "What will make it feel safe for yourself and your clients to return to the office?" The majority of the participants reported that a proven vaccine would be a catalyst to returning to the office $(82.2 \%$, $n=139)$. Moreover, $75.1 \%$ of the participants $(n=127)$ agreed with the following statement: "Until there is a safe and effective vaccine available, I don't see it as a safe and realistic option for me or my clients to be seen in person (conducting face-to-face sessions)." Participant GH explained: "I'm only open to doing that once we have the vaccine that actually works because my office is... I might be able to get some appropriate distance but the ventilation, you know, it just does not feel safe here." Only $34.3 \%$ of the participants $(n=58)$ reported being likely to return to the office before there is a proven vaccine.

Participants who agreed that if they return to the office before there is a proven vaccine, they will require their clients to wear masks, reported being less likely to return to the office before there is a vaccine in comparison to those who would not require their clients to wear a mask $(t=6.08$, $d f=155, p<0.001)$. Those who expressed feeling more comfortable working from home were also the ones who would require their clients to wear a mask when returning to inperson work, as opposed to those who would not require their clients to wear a mask ( $t=-2.39, d f=45.5, p=0.021)$.

More agreement was found with the statement "Until there is a safe and effective vaccine available, I don't see it as a safe and realistic option for me or my clients to be seen 
in person (conducting face-to-face sessions)." This was positively associated with participants' decisions not to return to the office: "I am not planning to return to the office in the foreseeable future" $(r=0.774, p<0.001)$.

The participants who reported being more likely to return to the office before there is a proven vaccine agreed less with the statement "I experience some stress or anxiety when I think about seeing clients in person" $(r=-0.368$, $p<0.001)$. The participants who reported being less anxious about seeing clients in person were more likely to return to the office before there is a vaccine.

\section{In-Person Therapy with Masks}

The participants had many mixed opinions on the idea of wearing a mask when meeting with clients in person. Seventy-four percent of the participants $(n=125)$ agreed that if they return to the office before there is a proven vaccine, they will wear a mask. Similarly, $71.6 \%$ of the participants $(n=121)$ agreed that they will also require their clients to wear masks. There was a positive association between the participants' agreement with the statement: "I will require clients to wear masks" and "I will wear a mask" $(r=0.871$, $p<0.001$ ), showing that the participants who will return to see clients in person and wear a mask will require their clients to wear one as well.

\section{Insurance Reimbursement}

Some of the participants interviewed described feeling at the mercy of the managed care system. "Insurance companies will be the first line of information, line of reference," stated participant DM, a licensed mental health counselor, when discussing making her decision to return to in-person work. "When they stop reimbursing," she argued, "is when people will be going back."

While only $45 \%$ of the participants $(n=76)$ reported being in-network with insurance providers, insurance reimbursement is considered a factor that may influence therapists' decisions on whether to return to seeing clients in person. Of the participants, $21.3 \%$ said they would return to the office to see clients if the insurance companies they work with cease reimbursing for online therapy $(n=36)$. Similarly, the participants who reported being in-network with insurance companies were more likely say they would return to the office to see clients if insurance companies no longer reimbursed for telepsychotherapy in comparison to those who were not in-network with insurance companies $(t=-4.65, d f=151, p<0.001)$. Participant DE shared the following:

I am hoping that health insurance companies are going to continue to put our health and mental health first... I think they have done a great job with covering clients' cost shares during this pandemic... my hope is that the insurance companies continue to make decisions that work so that people can continue to get the services that they need without endangering themselves.

So far, health insurance companies have been supportive of telepsychotherapy. However, if this changes, it may affect therapists' decisions to return to providing in-person therapy.

\section{Discussion}

At the time of writing, many therapists are continuing to provide telepsychotherapy, and some are doing so from their homes. When therapists will feel safe to return to seeing their clients in person again is unknown. However, the results from this study show that for most therapists, a blend of in-person and remote work would be the ideal approach to take in the future. Our findings show that the majority of therapists as well as their clients are not at all ready to return to in-person work due to safety concerns surrounding COVID-19. Nonetheless, they feel that there is a definite need for accessible therapeutic support and that it is still very important and necessary for them to provide telepsychotherapy.

Most therapists miss their in-person work but have adapted to the change; simultaneously, they reported that few of their clients have asked to return to in-person meetings. In fact, as reported by the participants, many of their clients have directly expressed their desire to continue with telepsychotherapy given its accessibility and convenience (no travel time, flexible schedules, can be done from the comfort of their own home, etc.). The majority of the therapists feel that telepsychotherapy has been as effective as in-person work, yet all agree that it is different and has required certain shifts to be made in the approach (e.g., it was important to take the time to build the therapeutic alliance, maintain eye contact, check in with clients more frequently, and have conversations about the process and getting comfortable).

Finally, a few factors were found to be associated with therapists' decisions to return to meeting clients in person. Therapists whose clients had asked about in-person sessions were more likely to return before there is a vaccine, and therapists who reported feeling less anxious were more prone to return to the office. Whether insurance companies continue to reimburse for telepsychotherapy was also associated with therapists' decisions to return to meeting clients in person or not. They also agreed that in order to return, there is a need for an effective vaccine and for them to feel safe without them or their clients having to wear a mask in sessions. 


\section{Practical Implications}

It is important that we advocate for continued insurance coverage of telepsychotherapy to ensure that both clients and therapists will be able to remain physically safe and feel emotionally secure as well. Requiring a premature return to in-person sessions before there is a proven vaccine and/or treatment for COVID-19 is likely to heighten anxieties and thereby create additional barriers to effective psychotherapeutic treatment. Nonetheless, there is a need to develop research that focuses on therapists who are currently providing in-person therapy in order to learn from them what factors had influenced their decisions to meet with their patients in person.

Effectively being forced to return to in-person work by insurance companies will create major barriers for populations that are at risk of contracting COVID-19 since they will have to decide between prioritizing their physical health or their mental health. This may further increase the gap between those who can afford private pay therapy and those who have to rely on insurance coverage. In order to inform insurance companies of the value of telepsychotherapy and advocate for its continued reimbursement, there is a need for future research to assess the effects of changes in insurance reimbursement on the provision of telemental health services.

At the moment, telepsychotherapy remains the preferred method, but it requires a different skill set in order to be as effective as in-person treatment. We recommend more training for therapists to maximize the efficacy of their remote work. We also recommend that therapists embrace opportunities to think outside of the box when providing psychotherapy during this unprecedented time. For example, they might consider holding physically distant sessions in outdoor spaces, which might be particularly effective when working with children. A creative shift in approaches to the therapeutic space may enable therapists to truly meet each individual client where they are and according to their needs, particularly for clients who are unable to receive the same benefits over the phone or video as they would face to face.

\section{Conclusions}

COVID-19 continues to shape the way therapists provide services. Although it remains unclear when therapists will return to providing traditional in-person therapy, the findings from this study show that several factors play a role in making this decision on an individual level. Overall, therapists' views on working from home, the lack of a proven vaccine, both clients' and therapists' satisfaction with telepsychotherapy, insurance companies' reimbursement for telepsychotherapy, and negative attitudes toward wearing masks during sessions affect whether therapists plan to return to traditional in-person psychotherapy in the near future. This current inquiry provides therapists with the opportunity to lean into the scientific data and find support as they make their own decisions on whether to return to the office as they work through this challenging time.

\section{References}

Aafjes van Doorn, K., Békés, V., \& Prout, T. A. (2020). Grappling with our therapeutic relationship and professional self-doubt during COVID-19: Will we use video therapy again? Counselling Psychology Quarterly. https://doi.org/10.1080/09515070. 2020.1773404

Backhaus, A., Agha, Z., Maglione, M. L., Repp, A., Ross, B., Zuest, D., \& Thorp, S. R. (2012). Videoconferencing psychotherapy: A systematic review. Psychological Services, 9(2), 111-131. https://doi.org/10.1037/a0027924

Békés, V., \& Aafjes-van Doorn, K. (2020). Psychotherapists' attitudes toward online therapy during the COVID-19 pandemic. Journal of Psychotherapy Integration, 30(2), 238-247. https:// doi.org/10.1037/int0000214

Békés, V., Aafjes-van Doorn, K., Prout, T. A., \& Hoffman, L. (2020). Stretching the analytic frame: Analytic therapists' experiences with remote therapy during COVID-19. Journal of the American Psychoanalytic Association, 68(3), 437-446.

Bell, C. A., Crabtree, S. A., Hall, E. L., \& Sandage, S. J. (2020). Research in counselling and psychotherapy post-COVID-19. Counselling and Psychotherapy Research, 20(3), 389-393.

Braun, V., \& Clarke, V. (2006). Using thematic analysis in psychology. Qualitative Research in Psychology, 3(2), 77-101.

Chherawala, N., \& Gill, S. (2020). Up-to-date review of psychotherapy via videoconference: Implications and recommendations for the RANZCP psychotherapy written case during the COVID-19 pandemic. Australasian Psychiatry, 28(5), 517-520.

Feijt, M., de Kort, Y., Bongers, I., Bierbooms, J., Westerink, J., \& Ijsselsteijn, W. (2020). Mental health care goes online: Practitioners' experiences of providing mental health care during the COVID-19 pandemic. Cyberpsychology, Behavior, and Social Networking, 16, 25-31.

Geller, S. (2020). Cultivating online therapeutic presence: Strengthening therapeutic relationships in teletherapy sessions. Counselling Psychology Quarterly, 2, 1-17.

Humer, E., Pieh, C., Kuska, M., Barke, A., Doering, B. K., Gossmann, K., \& Probst, T. (2020). Provision of psychotherapy during the COVID-19 pandemic among Czech, German and Slovak psychotherapists. International Journal of Environmental Research and Public Health, 17(13), 4811-4826.

Inchausti, F., MacBeth, A., Hasson-Ohayon, I., \& Dimaggio, G. (2020). Telepsychotherapy in the age of COVID-19: A commentary. Journal of Psychotherapy Integration, 30(2), 394-405. https://doi.org/10.1037/int0000222

Mitchell, E. (2020). "Much more than second best": Therapists' experiences of videoconferencing psychotherapy. European Journal for Qualitative Research in Psychotherapy, 10, 121-135.

Poletti, B., Tagini, S., Brugnera, A., Parolin, L., Pievani, L., Ferrucci, R., \& Silani, V. (2020). Telepsychotherapy: A leaflet for psychotherapists in the age of COVID-19. A review of the evidence. Counselling Psychology Quarterly, 2, 1-16. https://doi. org/10.1080/09515070.2020.1769557 
Roesler, C. (2017). Tele-analysis: The use of media technology in psychotherapy and its impact on the therapeutic relationship. Journal of Analytical Psychology, 62, 372-394.

Shigekawa, E., Fix, M., Corbett, G., Roby, D. H., \& Coffman, J. (2018). The current state of telehealth evidence: A rapid review. Health Affairs, 37(12), 1975-1982.

Shklarski, L., Abrams, A., \& Bakst, E. (2021). Navigating changes in the physical and psychological spaces of psychotherapists during Covid-19: When home becomes the office. Practice Innovations. https://doi.org/10.1037/pri0000138

Vostanis, P., \& Bell, C. A. (2020). Counselling and psychotherapy post-COVID-19. Counselling and Psychotherapy Research, 20(3), 389-393.
Wind, T. R., Rijkeboer, M., Andersson, G., \& Riper, H. (2020). The COVID-19 pandemic: The "black swan" for mental health care and a turning point for e-health. Internet Interventions, 20, 1-2.

Wright, J. H., \& Caudill, R. (2020). Remote treatment delivery in response to the COVID-19 pandemic. Psychotherapy and Psychosomatics, 89(3), 30-132.

Publisher's Note Springer Nature remains neutral with regard to jurisdictional claims in published maps and institutional affiliations. 